\title{
Construcción de la identidad racial: una mirada desde la familia negra cartagenera*
}

KRISTELL VILLARREAL BENÍTEZ ${ }^{* *}$ kavbfolk@hotmail.com

Recepción: 10 de febrero de 2015

Aprobación: 02 de abril de 2015

Forma de citar este artículo: Villarreal Benítez, K. (2016). Construcción de la identidad racial: una mirada desde la familia negra cartagenera. Cuadernos de Lingüística Hispánica, (27), 19-31.

* Artículo de investigación

** Profesional en lingüística y literatura de la Universidad de Cartagena, Colombia; integrante del grupo de investigación TEXCULTURA (Texto, discurso y cultura en el Caribe colombiano), en la línea de discurso, reconocimiento e identidad. 


\title{
Resumen
}

El propósito de este artículo es exponer el rol de la familia en la reproducción del racismo y la confrontación por parte de sus miembros ante la discriminación racial. Los discursos socializadores emitidos en el contexto de la familia suelen contener información respecto a cuestiones identitarias, asuntos referentes a la cultura, la raza y la sociedad donde se desenvuelve la familia; respondiendo a ello, se encontró que la familia negra de Cartagena reproduce ideas racistas que circulan en los discursos de la sociedad cartagenera.

Palabras clave: Confrontación, discriminación, familia, racismo y reproducción.

\section{Racial identity construction: a glance from the perspective of the black families of Cartagena.}

\begin{abstract}
The purpose of this article is to discuss the role of the family in the reproduction of racism and the way in which its members are confronted by racial discrimination. The socializing discourse given in the context of family often carries information about matters of identity, as well as issues related to the culture, race and society where the family is involved. By analizing this context, we found that black families in Cartagena reproduce a large amount of the racist ideas common in the city.
\end{abstract}

Key words: Confrontation, discrimination, family, racism and reproduction of discourse. 


\title{
Construction de l'identité raciale: un regard dès la famille noire à Cartagena
}

\section{Résumé}

Le propos de cet article est celui d'exposer le rôle de la famille dans la reproduction du racisme et la confrontation de la part de ses membres, face à la discrimination raciale. Les discours socialisateurs faits dans le contexte de la famille, contiennent généralement de l'information par rapport aux questions identitaires, des affaires référant à la culture, la race et la société, où la famille se développe. Répondant à ceci, nous trouvons qu'une famille noire à Cartagena, reproduit des idées racistes qui circulent dans les discours de la société de Cartagena.

Mots clés: confrontation, discrimination, famille, racisme et reproduction.

\author{
Construção da identidade racial: \\ uma mirada desde a família negra cartagenera
}

\section{Resumo}

0 propósito deste artigo é expor o rol da família na reprodução do racismo e a confrontação por parte de seus membros ante a discriminação racial. Os discursos socializadores emitidos no contexto da família costumam conter informação a respeito a questões identitárias, assuntos referentes à cultura, à raça e à sociedade onde se desenvolve a família; respondendo a esta questão, encontramos que a família negra de Cartagena reproduz ideias racistas que circulam nos discursos da sociedade cartagenera.

Palavras chave: confrontação, discriminação, família, racismo e reprodução. 


\section{Introducción}

La familia puede definirse como "un grupo o sistema compuesto por subsistemas que serían sus miembros y a la vez integrada a un sistema mayor que es la sociedad" (Ares, 1990, p. 77). Consecuente con esta definición, se asume que la familia al estar relacionada directamente con la sociedad produce discursos legitimando, señalando o reproduciendo representaciones tanto positivas como negativas acerca de los sujetos que conforman los diferentes grupos sociales.

Berger \& Luckmann (1995) establecen el concepto de socialización primaria, los autores la definen como aquella "que el individuo atraviesa en la niñez, por medio de ella se convierte en miembro de la sociedad" (p. 166). La socialización primaria se lleva a cabo en el entorno familiar y ayuda a que los niños reconozcan los roles, los aprehendan y los acepten; a partir de ello empezarán a construir sus identidades de forma autónoma, ya que serán capaces de reconocer al otro. Sin embargo, hay que resaltar que esa identidad se construirá de acuerdo a los conocimientos que han obtenido sus agentes socializadores.

Asumir que dentro de los discursos familiares solo se tienen en cuenta temáticas tales como normas, hábitos, costumbres o tradiciones, es dejar de lado que la familia es un espacio que se encuentra articulado con una estructura social y que, por ende, también posee características y comparte ideas que son reproducidas y aceptadas en la sociedad. Se encuentra, entonces, que los discursos emitidos por padres, abuelos o tíos vienen cargados de estereotipos culturales y raciales; diferenciaciones por cuestiones de género o religión.

Con lo anterior, se puede afirmar que aquellas inequidades que se evidencian a nivel macrosocial tienen repercusiones palpables, gracias a los agentes sociales, dentro de los pequeños sistemas que reconocemos como hogares y que los individuos que sustentan ese sistema ayudan a que la desigualdad se perpetúe.

La familia como eje central de la estructura social se consolida como un espacio necesario para comprender la reproducción del racismo dentro de la sociedad cartagenera; 
elementos propios del contexto sociocultural como la estructura de la familia o su estatus social y económico, son fundamentales para entender todo el proceso de perpetuación del racismo.

Hablar de la familia en general ya es en sí asomarse en un terreno con un tratamiento difícil. Al momento de hablar de la familia en un contexto determinado como el de Cartagena de Indias aparecen conceptos y valores un tanto arduos de comprender, ya que cada una de sus características se relaciona directamente con elementos sociales y un pasado histórico cuyos vestigios aún son visibles. Encontramos, entonces, que los tipos de familia, su constitución, los roles de los integrantes, etc. son aspectos que se encuentran regidos por normas socialmente establecidas.

De acuerdo a los eventos narrados por las personas entrevistadas, se pudo observar que hubo una fluctuación de conocimientos y de representaciones acerca de la raza, lo cual ayudó a vislumbrar los primeros "bosquejos" de la identidad racial de los informantes. La investigación realizada buscó explicar cómo las familias negras enfrentaban el fenómeno del racismo y cómo le enseñaban a sus hijos a confrontarlo.

\section{Marco teórico}

Esta investigación se encuentra enmarcada dentro de la perspectiva teóricometodológica del análisis crítico del discurso. Se tuvieron en cuenta diversas nociones tales como la de racismo cotidiano (Essed, 1991), el racismo percibido (Mellor, 2003; Merino, 2007); la idea de la narrativa como forma de expresar la experiencia (Bruner, 1991; Labov, 1988) y la tipología de familia negra (Boykin \& Toms, 1985).

\subsection{Socialización}

Los elementos que determinan los rasgos de la personalidad, la identidad de los individuos, el reconocimiento como ser social que marcará el punto de partida del yo y el inicio del tú, el auto-reconocimiento del niño en un grupo social específico, etc., comienzan a construirse al interior de la familia, de allí que la socialización sea entendida como:

El proceso por cuyo medio la persona humana aprende e interioriza, en el trascurso de su vida, los elementos socioculturales de su medio ambiente, los integra a la estructura de su personalidad, bajo la influencia de experiencias y de agentes sociales significativos, y se adapta así al entorno social en cuyo seno debe vivir (Rocher, 1990, p. 133) 
En las sociedades actuales, la socialización no corresponde únicamente a la familia. Existen instituciones como la escuela o la iglesia donde el niño afianza lo aprendido en el hogar o lo modifica de acuerdo con la relación que establezca con su entorno y con el conocimiento ofrecido en este.

Si se asume que la socialización es un proceso de aprendizaje por parte del niño y de enseñanza por parte del padre tendremos que es algo que toma lugar en el marco discursivo, ya que, la mayor parte de las interacciones que se dan entre los individuos están mediadas por el lenguaje. El uso del discurso para expresar y trasmitir conocimientos al niño se ve afectado por todas aquellas ideologías que maneje el sujeto socializador, de allí que todos los sujetos socializados construyan un marco conceptual "coherente" con el contexto social.

\subsection{Socialización racial e Identidad racial.}

Racial socialization is the primary vehicle of cultural transmission for African American families, steeped in a tradition of resistance to oppression and embedded in "conversations and actions that communicate to [our] children how to survive with dignity and pride in a racist world. (Stevenson, Davis \& Abdul-Kabir, 2001, p. 46).

La socialización racial es un proceso que lleva a cabo la familia Negra, puesto que, una socialización que no contenga mensajes positivos acerca de su identidad racial, su identidad personal y su autoestima no permitirá que los niños afronten el fenómeno del racismo de una forma equilibrada y reconociendo sus valores culturales como un componente positivo.

La socialización racial es conceptualizada por muchos investigadores como el discurso socializador más significativo que se brinda al interior de las familias afrodescendientes, porque prepara a los niños física y mentalmente para hacer frente a un problema que se permea en todas las esferas de la vida social. Al respecto, Marshall (1995) expresa "it prepares African American children to be psychologically and physically healthy in spite of racism and discrimination". En esencia, no es una cuestión de que el niño "subsista" en un contexto racista, sino que se desarrolle saludablemente y sin mayores secuelas a lo largo de su vida.

Lo que se aprende en el hogar puede articularse de tal forma que permita la construcción de una identidad racial, sea positiva o negativa en el sujeto socializado; al respecto, Lionel Scott (2003) dice que "Black identity that is "unassailable" is the mostsignificant strategy for resistance to racial oppression and hence is requisite for African 
American children and adolescents" (p. 523). Sin embargo, esta identidad racial de la que habla Scott es la que Thomas \& Speight (1999) definen como identidad racial positiva "has been empirically linked to increased psychological adaptation and functioning, increased self-esteem".

De acuerdo al tipo de identidad que construya, el sujeto se establecerá las respuestas que proporcionará al asumir y experimentar conflictos de índole racial en diferentes espacios.

\section{Metodología propuesta}

\subsection{Datos y Participantes}

La recolección de los datos fue realizada en dos momentos temporales diferentes; las primeras entre diciembre de 2006 y abril de 2007 por la doctora Elisabeth Cunin y el segundo grupo de entrevistas entre junio y agosto de 2009 por integrantes del grupo de investigación TEXCULTURA. Así pues, fueron realizadas 20 entrevistas en total.

Las entrevistas se caracterizan por seguir una secuencialidad en cuanto a las etapas vitales de los sujetos entrevistados. Por esa razón, se pudo ahondar considerable en la etapa vital de la infancia, los informantes enfatizaron diversos aspectos relevantes de la crianza que tuvieron en sus hogares. Se realizó un proceso de digitalización y de transcripción de las entrevistas teniendo en cuenta el modelo construido por el grupo Val.Es.Co.

Los informantes son personas negras, cartageneras, profesionales, integrantes de movimientos que luchan contra el racismo en la ciudad de Cartagena; son mayores de edad y todos se encuentran relacionados con FUNSAREP. Se utilizó la técnica de muestreo "bola de nieve" para conseguir más personas que quisieran participar. Los informantes debían cumplir con una serie de características fundamentales para poder ser entrevistados, es decir, "asumirse como afrodescendientes, tener conocimiento de la problemática racial de Cartagena, ser miembro de organizaciones políticas o académicas donde se reflexione al respecto." (De la Hoz, Severiche \& Correa, 2014, pp. 121-122).

A las personas entrevistadas se les dio un seudónimo y de esa forma se ha buscado proteger su identidad e integridad. Se realizaron fichas descriptivas de la situación de entrevista, las cuales contenían información básica como la edad, sexo, profesión, etc. La finalidad de esto fue recopilar información que permitiera descifrar, en algún punto de la entrevista, aquellos eventos relacionados directamente con las características sociodemográficas que poseía el sujeto entrevistado. 
Para la realización del análisis de los datos en esta investigación se dividieron todas aquellas experiencias y se tuvieron en cuenta las que fueron vividas, las que fueron presenciadas y las que fueron experimentadas por terceros. Se recurrió a un análisis minucioso de las entrevistas a través de un software llamado Antconc, el cual permitió focalizar todos los microrrelatos que se enmarcaban en el hogar.

\section{Análisis}

En un primer momento, se aislaron los segmentos que contenían tópicos relacionados directamente con el hogar; se encontraron un total de 180 segmentos discursivos que hacían referencia a situaciones racistas al interior del hogar. Los datos conseguidos se caracterizan por tener dos estructuras básicas para relatar los eventos que tuvieron lugar en el hogar; en primera instancia tenemos la estructura narrativa:

(1) "Por lo menos mi papá yo considero que es el personaje principal del racismo que yo conozco en él siempre siento jehm! Ese hecho de no ir $\uparrow$ a los lugares donde vas a ser excluido, o sea evitar de asistir los lu, a lugares donde no vas a ser recibido por ser Negro (...)" (Miladys, 2009).

Las narraciones obtenidas manifiestan eventos poco usuales que no expresan adecuadamente lo que se asume por familia. En segundo lugar tenemos las descripciones:

(2) “(...) otro personaje que yo conozco así bien racista es mi ABUELA MATERNA, por parte de mi mamá. Mi abuela era mella, delgadita, Negra, eh con ciertas facciones del Negro muy marcadas $(())$ ciertas características, nariz achacada, eh pelo súper crespo. Pero mi abuela discriminaba todo, para ella todo el mundo era feo, era Negro, era bembón (...)” (Miladys, 2009).

Las narraciones de los entrevistados se caracterizan por no hacer un resumen de lo que se narrará, sino que se remiten directamente a la orientación y abordan la contextualización de los actores, del evento en particular, y de aspectos como el lugar y la época. Los entrevistados desarrollan la complicación y dan a conocer qué sucedió.

Los informantes construyeron narraciones sencillas que contenían un resumen, una orientación y una complicación. Cabe resaltar que solo unos pocos entrevistados realizaron una evaluación de los eventos, y al momento de hacerlo utilizaron mucho las repeticiones consiguiendo hacer énfasis y dar explicaciones sobre el evento narrado.

En un segundo momento, al terminar la segmentación de las narraciones y realizar una lectura minuciosa, se notó la repetición de unas macro-proposiciones tales como: "hay que mejorar la raza" o "búscate a un blanco". Estas, al ser tomadas como el tema central del relato, enseñan que son portadoras de las representaciones del tema racial para 
la familia de los informantes. También se encontraron mensajes como: "tienes que lavar la raza" o "debes adelantar la raza", los cuales fueron utilizados para socializar en la mayoría de los casos a las mujeres entrevistadas. ${ }^{1}$

\section{Los resultados}

Realizar estudios acerca de la familia implica introducirse en un terreno frágil, ya que es un espacio construido sobre la idea de lo íntimo. Los informantes al momento de contar acerca de los eventos que tuvieron lugar al interior de su núcleo familiar, relataron cada uno de los cuestionamientos acerca de la raza que evidenciaban los padres, abuelos, tíos o familiares que cumplían un rol socializador al interior de la familia.

(3) "Ellos no sabían que eran racistas sino que ellos creían que era normal, que había que decir eso, que... que yo soy Negro, que yo tengo que salirme de, dejarme de ser Negro, ya, para ellos era malo ser Negro." (Pedro, 2006)

(4) "Mi mamá..., cuando yo era una adolescente me decía, más ya yo estaba identificada con quién era yo. Me decía, este, mija que no te vaya a pasar como a mí, búscate un hombre blanco, búscate un hombre bonito, para que laves la raza, ya aquí hay mucho Negro..., y a los blancos les gustan las Negras, búscate un blanco bien bonito" (Ana, 2006).

Los padres enseñan de aquello que aprendieron. Ellos brindan referencias acerca del mundo que está afuera, ofreciéndole a los niños un grupo social con el que representarse, valores que adoptar, actitudes que tener y patrones de comportamientos que asumir.

Si bien, la familia es conceptualizada como aquella institución encargada de moldear factores identitarios, no siempre resaltan o contribuyen a la aceptación de valores raciales y étnicos. En el caso de la familia negra cartagenera, se encuentra pues que se les enseña a sus hijos que la forma de hacer resistencia frente al racismo es través del camuflaje de todos aquellos rasgos físicos y culturales que los caracterizaban como personas Negras.

Essed (1991) dice que:

knowledge of the problematization of Blacks reflects the way these ideological notions rationalize the marginalization of Blacks traditions and values as well as the exclusion of Blacks from Access to material and nonmaterial recourse, on the one hand, and on the other hand, the suppression of Blacks in order to contain them in relations of dominance (p. 114).

1 De los 10 informantes de sexo femenino, se encontró que 6 fueron socializadas con estos tipos de mensajes. 
Las personas en Cartagena que se han enfrentado al racismo y han aprendido acerca de este fenómeno, procuran contrarrestar cada uno de los procesos de racismo (marginalización, problematización y sometimiento), problematizándose a sí mismos.

En la investigación se encontró pues, que en la familia Negra cartagenera la socialización racial se fundamenta en tres ítems principales:

- La valoración que realiza el sujeto socializador de todos los eventos discriminatorios vividos.

- La problematización de todos aquellos elementos que lo presentaron como un sujeto diferente y lo expusieron a la discriminación.

- Todas aquellas respuestas que dieron los agentes socializadores a los eventos discriminatorios y que, posteriormente, pasaron a ser conocimiento socializador, es decir, conocimiento que se les enseña a los hijos para que adquieran esas mismas actitudes.

Vemos que al interior de los hogares se construyen formas de enfrentar el racismo positiva o negativamente; partiendo de esto, las formas de avistar a los individuos de raza Negra difiere de acuerdo al tipo de socialización que han recibido los agentes socializados y el tipo de identidad que han construido en relación con los conocimientos interiorizados.

Otro punto que fue notorio durante el análisis de los datos fue el proceso de formación de familia en Cartagena, el cual sugiere una serie de singularidades. A través de las entrevistas se hicieron visibles dos situaciones complejas; la construcción de hogares entre iguales y la construcción de los mismos entre parejas interraciales. Ambos eventos conllevan a una serie de respuestas por parte del núcleo familiar en la que el individuo está inserto. Así pues existen uniones que se consideran aceptadas y otras que se plantean como conflictivas.

Respecto a lo anterior, los informantes reportaron lo siguiente:

(5) "Sin embargo, yo siembre (sic) noté, inclusive en el seno de mi familia donde eran las personas eh, de de (sic) lo que es el emblanquecimiento sí. El el afán de dejar de ser Negro" (Carlos, 2006).

"Carlos" afirma que su familia quería dejar de ser Negra y para ello recurrieron a métodos que les permitieran asemejarse al grupo Blanco. Una de las formas más usuales de blanqueamiento es conseguir una pareja Blanca con el propósito de "blanquearse". 
(6) "Mi casa no hay ni medio blanco, en mi familia tú ni consigues ni uno ni así. Los que son más claritos son las nuevas generaciones, pues mi hermano se casó con una muchacha que es comooo tu color pelo indio, mucho más clara y mi hermana que tiene el marido que es cachaco. Te estoy hablando de niños de dos años/ y de seis años, pero de la mía pa atrás, 15 años pa" atrás. No busques ningún blanco que no lo vas a encontrar (RISAS)" (Adolfo, 2009).

La respuesta de los integrantes de la familia estaba sujeta al grupo racial al que pertenecían; los nuevos hogares conformados por dos miembros interraciales donde el hombre era Negro y la mujer Blanca, se enfrentaron a situaciones problemáticas con los familiares.

(7) "Era clara bastante y el muchacho, o sea, amigo mío, era morenito, pelo cuscui y tal, ya! Entonces ¿¡cómo vas a dañar la familia!? (()) y mandaron a la muchacha, la mandaron pa'el pueblo" (Juan, 2009).

(8) "Cuando yo era novio de mi mujer - de mi segunda esposa no - eh... nunca me dejaron entrar a su casa porque yo era Negro, y el gran temor de la familia de ellos es que nuestros hijos iban a ser Negros" (Orlando, 2006).

El eje del conflicto se hallaba al interior de la familia Blanca, la cual no permitía ni daba el aval para que una persona Negra hiciera parte de su "grupo familiar". Sin embargo, para las personas que se encuentran en evidente desventaja en el contexto social de Cartagena por factores raciales, la inclusión de personas Blancas es un elemento decisivo en la adquisición de un rol significativo en Cartagena.

Al establecerse relaciones entre personas de la misma raza (Negros con Negros), el eje conflictivo era liderado por aquellos familiares que conceptualizan "el mejoramiento racial" y que construían a las personas Negras como individuos que obstruían la posibilidad de un progreso fenotípico y social. Las situaciones narradas por los informantes podían ser asumidas como procesos de discriminación racial, sin embargo, resultaba improcedente dejar de lado la intencionalidad de los sujetos que desempeñaban el rol de agentes socializadores.

Los padres de nuestros informantes son personas Negras cuyas experiencias les habían indicado la manera propicia para amoldarse y responder a eventos con matices discriminatorios que se daban en la ciudad. De acuerdo a lo anterior, la socialización es "the preparation of children to accept adult roles and responsibilities in society through the teaching and learning of conventional beliefs, values, and patterns of behavior" (Boykin \& Toms, 1985). 
Es conveniente aclarar que la socialización que se da en las familias negras de Cartagena funciona como un puente conductor de conocimientos experienciales, adquiridos en algún momento por los padres y transmitidos por estos mismos a los hijos. Dichos conocimientos permiten la creación y el fortalecimiento de las competencias que requieren los sujetos Negros para entrar con una "dinámica positiva" en esta sociedad en particular.

\section{Conclusiones}

Aún falta terreno que recorrer en los estudios del racismo en relación con la familia. Sin embargo, estos fueron los primeros esbozos que permitirán el acercamiento a los eventos que tienen lugar en la ciudad.

En este artículo se da a conocer la existencia de múltiples mensajes socializadores con contenido racial discriminatorio. La información obtenida a partir del análisis de los datos se encontraba inmersa en contextos con diversos grados de complejidad, ya que en ocasiones hacía referencia a un miembro específico de la familia, pero en otras ocasiones se refería al grupo negro en general.

La presencia de ideologías racistas en los discursos familiares dio cuenta de la supervivencia del racismo a nivel microsocial y su presencia constante en la cotidianidad de los individuos. Por otra parte, fue notoria la prevalencia de las categorías sociales, raciales y de todos aquellos estereotipos e ideas que circulan a nivel macro.

En cuanto a la identidad racial, se puede concluir que los padres, en el afán de querer que sus hijos encajen en la sociedad cartagenera, desglosan los discursos racistas y aprehenden aquello que les es útil al momento de socializar, de esta forma enseñan cuáles son las actitudes más convenientes para que sus hijos no sean víctimas de exclusión.

Si bien los objetivos de los padres no incluyen la perpetuación de la discriminación, sus acciones si consiguen fomentar la construcción negativa de la identidad racial del niño. Como consecuencia, no se promueve la aceptación de los elementos físicos, étnicos y culturales que los caracterizan dando así continuidad al racismo desde el interior de la institución más importante en la sociedad. 


\section{Referencias bibliográficas}

Ares, P. (1990). Mi familia es así. La Habana: Ed. Ciencias Sociales.

Berger, P. L., y Luckmann, T. (1995). La construcción social de la realidad. Buenos Aires: Amorrortu editores.

Boykin, A. W., y Toms, F. D. (1985). Black child socialization: A conceptual framework. En: H. P. McAdoo y J. L. (Ed.). Black children: Social, educational, and parental environments. (pp. 33-51). Thousand Oaks, CA: Sage Publications.

Bruner, J. (1991). The narrative construction of reality. CriticalInquiry, 18(1), 1-21.

De la Hoz, D., Severich, D., y Correa, C. (2014). Formas de aproximarse a la "palabra de los otros": la entrevista etnográfica. En: C. I. Fonseca Mendoza (comp.), Espacios de discriminación racial en Cartagena. (pp. 117-133). Cartagena de Indias, Colombia: Autoreseditores.com.

Essed, P. (1991). Understanding everyday racism. An interdisciplinary theory. Newbury Park, CA: SAGE publications.

Labov, W. (1988). La transformación de la experiencia en sintaxis narrativa (M. de Cobo, trad.) Universidad del Valle: Centro de traducciones.

Marshall, S. (1995). Ethnic socialization of African American children: Implications for parenting, identity development, and academic achievement. Journal of Youth and Adolescence, 24(4), 377-396.

Mellor, D. (2003). Contemporary racism in Australia: The experiences of Aborigenes. Personality and Social Psychology Bulletin, (29); 474. Recuperado de http://psp.sagepub.com/cgi/ content/abstract/29/4/

Merino, M E. (2007). El discurso de la discriminación percibida en Mapuches de Chile. Revista Discurso \& Sociedad, 1(4), 604-622. Recuperado de www.dissoc.org

Rocher, G. (1990). Introducción a la Sociología general. Barcelona, España: Herder.

Scott, L. D. (2003). The Relation Of Racial Identity And Racial Socialization To Coping With Discrimination Among African American Adolescents. Journal of Black Studies, (33), 520538. Doi: $10.1177 / 0021934702250035$

Stevenson, H. C., Davis, G., Abdul-Kabir, S. (2001). Stickin' to, watchin' over, and gettin' with: An African American parent's guide to discipline. San Francisco, StatesUnited of America: Josey-Bass. 